\title{
Theme 3: Non-invasive management of (recurrent) venous thromboembolism (VTE) and post thrombotic syndrome (PTS)
}

Citation for published version (APA):

ten Cate-Hoek, A. J., Weitz, J. I., Gailani, D., Meijer, K., Philippou, H., Bouman, A. C., Whitney Cheung, Y., van Mens, T. E., Govers-Riemslag, J. W., Vries, M., Bleker, S., Biedermann, J. S., Stoof, S. C. M., \& Buller, H. R. (2015). Theme 3: Non-invasive management of (recurrent) venous thromboembolism (VTE) and post thrombotic syndrome (PTS). Thrombosis Research, 136(Supplement 1), S13-S18. https://doi.org/10.1016/j.thromres.2015.07.036

Document status and date:

Published: 01/09/2015

DOI:

10.1016/j.thromres.2015.07.036

Document Version:

Publisher's PDF, also known as Version of record

\section{Document license:}

Taverne

Please check the document version of this publication:

- A submitted manuscript is the version of the article upon submission and before peer-review. There can be important differences between the submitted version and the official published version of record.

People interested in the research are advised to contact the author for the final version of the publication, or visit the DOI to the publisher's website.

- The final author version and the galley proof are versions of the publication after peer review.

- The final published version features the final layout of the paper including the volume, issue and page numbers.

Link to publication

\footnotetext{
General rights rights.

- You may freely distribute the URL identifying the publication in the public portal. please follow below link for the End User Agreement:

www.umlib.nl/taverne-license

Take down policy

If you believe that this document breaches copyright please contact us at:

repository@maastrichtuniversity.nl

providing details and we will investigate your claim.
}

Copyright and moral rights for the publications made accessible in the public portal are retained by the authors and/or other copyright owners and it is a condition of accessing publications that users recognise and abide by the legal requirements associated with these

- Users may download and print one copy of any publication from the public portal for the purpose of private study or research.

- You may not further distribute the material or use it for any profit-making activity or commercial gain

If the publication is distributed under the terms of Article $25 \mathrm{fa}$ of the Dutch Copyright Act, indicated by the "Taverne" license above, 
Full Length Article

\title{
Theme 3: Non-invasive management of (recurrent) venous thromboembolism (VTE) and post thrombotic syndrome (PTS)
}

\author{
Arina J. ten Cate-Hoek ${ }^{\mathrm{a}, \mathrm{b}, *}$, Jeffrey I. Weitz ${ }^{\mathrm{c}}$, David Gailani ${ }^{\mathrm{d}}$, Karina Meijer ${ }^{\mathrm{e}}$, Helen Philippou ${ }^{\mathrm{f}}$, \\ Annemieke C. Bouman a,b , Y. Whitney Cheung ${ }^{g}$, Thijs E. van Mens ${ }^{g}$, Jose W. Govers-Riemslag ${ }^{a}$, Minka Vries ${ }^{\text {a }}$, \\ Suzanne Bleker ${ }^{\mathrm{g}}$, Jossi S. Biedermann ${ }^{\mathrm{h}}$, S. Carina M. Stoof ${ }^{\mathrm{h}}$, Harry R. Buller ${ }^{\mathrm{g}}$ \\ ${ }^{a}$ Maastricht University Medical Center, Laboratory for Clinical Thrombosis and Hemostasis, Cardiovascular Research Institute Maastricht (CARIM), Netherlands \\ b Thrombosis Center, USA

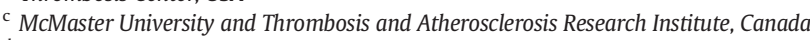 \\ d Vanderbilt University, Department of Pathology, Microbiology and Immunology, Nashville, USA \\ e University of Groningen, University Medical Center Groningen, Department of Hematology, Netherlands \\ ${ }^{\mathrm{f}}$ University of Leeds, Division of Cardiovascular and Diabetes Research, The LIGHT Labs, Leeds, UK \\ ${ }^{g}$ University of Amsterdam, Academic Medical Center, Department of Vascular Medicine, Netherlands \\ ${ }^{\mathrm{h}}$ Erasmus University Medical Center, Rotterdam, Department of Hematology, Netherlands
}

\section{Background}

Over the last 30 years, conservative management of VTE has focused mainly on the prevention of recurrent thrombotic events. The prevention of long-term complications of venous thromboembolism (VTE), such as post thrombotic syndrome (PTS), has gained less attention. The quest for anticoagulant drugs that are equally effective as vitamin $\mathrm{K}$ antagonists (VKAs) and that are easier to manage has concluded in a favorable manner. For treatment of VTE, the non-vitamin $\mathrm{K}$ antagonist oral anticoagulants (NOAC) are non-inferior to the VKAs with respect to efficacy and are at least as safe if not safer. However, the NOACs do not eliminate the risk of bleeding and gastrointestinal bleeding occurs more frequently with some of the NOACs than with VKAs, particularly in the elderly. This is an important issue because the risk of bleeding makes long term anticoagulation for secondary VTE prevention less attractive. To address this problem, efforts are underway to identify anticoagulants that are as effective as current treatments (VKAs and NOACs) but produce less bleeding. Strategies that target factor XII or factor XI are particularly promising and if agents can be identified that attenuate the risk of thrombosis without affecting hemostasis, the issue of recurrent thrombosis could easily be solved because patients could continue therapy as long as needed, assuming there are no off -target side effects. The risk of complications of DVT such as PTS, which occurs in $20-50 \%$ of patients, is, amongst others, associated with poor early control of anticoagulant treatment and could possibly be reduced with more stable levels of anticoagulation.

\footnotetext{
* Corresponding author at: Department of Internal Medicine, laboratory of Clinical Thrombosis and Haemostasis, Maastricht University Medical Centre +, PO Box 616, 6200 MD Maastricht, The Netherlands.

E-mail address: arina.tencate@maastrichtuniversity.nl (A.J. ten Cate-Hoek).
}

\section{Past Achievements}

Antithrombotic therapy with VKAs is highly effective and has been successfully used for over 60 years. The two major drawbacks of VKAs are the risk of serious bleeding, such as intracranial bleeding, which can occur even when the international normalized ratio (INR) is in the therapeutic range, and the requirement for routine coagulation monitoring because of the numerous drug-drug and drug-food interactions. These drawbacks were the incentive for the development of new anticoagulants that could be given in fixed doses without routine coagulation monitoring and were safer than VKAs. The NOACs fulfill these goals; they are easier to manage than VKAs, and are at least as effective and produce less intracranial bleeding.

Long-term aspirin is widely used for secondary prevention of arterial thrombosis. Recently, aspirin has been compared with placebo for secondary prevention of VTE with the expectation that its benefit-to-risk profile would make it an attractive alternative for patients unwilling or unable to take VKAs long-term. A recent meta-analysis of two such studies showed that compared with placebo, aspirin reduced the risk of recurrent VTE by $32 \%$ and was associated with a low risk of bleeding. The role of statins and contact activation inhibitors for prevention of recurrent VTE remains to be established but initial clinical data are promising.

The long-term complications of deep vein thrombosis (DVT) and pulmonary embolism (PE), such as PTS and chronic thromboembolic pulmonary hypertension (CTEPH), have been understudied. The Villalta score is the preferred method for diagnosis of PTS, but there is room for improvement. Although compression therapy was the mainstay for prevention of PTS, a recent double blind trial found no effect with early application of graded compression stockings. Nonetheless, compression stockings continue to be used to manage PTS symptoms. Studies into the pathophysiology of PTS have identified potential mechanisms, and further investigation is needed to determine whether this 
mechanistic information will translate into new targets for drugs to prevent this debilitating complication.

\section{Role of Statins in venous thromboembolism (VTE)}

[Harry R. Büller, Jossi S. Biedermann]

During 6-12 months of treatment with NOACs, the risk of recurrent VTE is approximately 2\% [1]. After stopping NOACs, the rate of recurrent VTE at 6 and 12 months is $6.2 \%$ and $10.7 \%$ respectively [2,3]. Hence, both during and after anticoagulant therapy there is an unmet need to further reduce the risk of recurrent disease. It is likely that targeting other important biological pathways outside coagulation, most notably the (vascular) inflammatory pathway, is most promising, particularly if such interventions do not increase the risk of bleeding.

Statins have diverse effects, including modulation of almost all of the components involved in thrombosis, i.e. platelets, endothelium, tissue factor and other coagulation factors [4,5]. The net result of statin administration is invariably a reduction in thrombogenicity.

Numerous cohort studies [6] and population studies [7-9] suggest that statin therapy may dose dependently reduce the risk of recurrent VTE by $25-40 \%$. There is only one randomized trial, which compared rosuvastatin with placebo for primary VTE prevention in subjects with increased levels of C-reactive protein [10]. Although the absolute rates were low, this study reported a $43 \%$ reduction in VTE.

Capitalizing on developments in arterial thrombosis, we can expect an explosion of clinical data with agents that target local and systemic inflammation. These include old drugs like, methotrexate and colchicine, as well as new agents that inhibit interleukin (IL)-1B, IL6, tumor necrosis factor (TNF) and lipoprotein-associated phospholipase A2 [11]. The time has come in the VTE field to study drugs that control inflammation both from a basic and a clinical perspective.

Consensus:

a. Current treatment for VTE needs improvement, mainly in secondary prophylaxis.

b. The role of statins needs to be elucidated in clinical trials.

c. The ideal trial design would be direct start in patients with a first unprovoked VTE, with imbedded nested mechanistic studies

d. The best outcome measure is: recurrent VTE, but arterial events and PTS should also be assessed as combined outcomes

e. As mono-therapy, statins should only be considered for secondary VTE prevention in patients who refuse or cannot tolerate anticoagulants or aspirin.

\section{Aspirin for VTE prevention}

[Karina Meijer, Minka Vries]

In recent years, the utility of aspirin for primary and secondary VTE prevention has been studied in clinical trials and has become a subject of heated debate. Concerning thromboprophylaxis after major orthopedic surgery, those who deem 'fatal PE' as the only relevant outcome favour aspirin. For this outcome, the Pulmonary Embolism Prevention trial showed an advantage of aspirin over placebo [12]. Those who favour 'all VTE' as the outcome prefer low molecular weight heparin (LMWH) over aspirin. The 2012 ACCP guidelines recommend all pharmacological means, including aspirin, over no prophylaxis, but LMWH was recommended over other agents (Grade 2B/2C) [13]. However, interest was rekindled after Anderson et al. showed that after an initial 10-day course of LMWH, extended treatment with aspirin was noninferior to LMWH [14]. We discussed these data, and concluded that further studies in this field are of low priority because there is no unmet clinical need; LMWH and the NOACs offer safe, effective and convenient means of prophylaxis.

For secondary prevention of VTE after a first non-provoked episode, the combined results of the WARFASA and ASPIRE studies demonstrate that compared with placebo, aspirin reduces the risk of recurrent VTE by $32 \%$ (hazard ratio [HR], 0.68; 95\% confidence interval [CI], 0.51-0.90)
[15]. However, the important question is how aspirin compares with VKAs or NOACs to determine whether aspirin is a better option than conventional or new anticoagulants. Direct comparisons are not available at this point - although a study comparing aspirin with rivaroxaban is ongoing - but a published network meta-analysis summarizes all of the indirect evidence [16]. This evidence was discussed, and we reached consensus that for the majority of patients, an anticoagulant would offer a better trade-off between efficacy and safety/convenience than aspirin.

A possible patient category that would benefit from aspirin would be those with a low-to-moderate risk of recurrence. Before studies into these patient categories should be initiated, however, more precise recurrence prediction tools are needed.

Three other interesting points were raised. First, the mechanism by which aspirin prevents VTE is unknown. It is possible that the antiinflammatory or other protective effects of aspirin predominantly occur when the endothelium is already damaged or activated, such as after surgery or DVT. Second, because patients with cancer have platelet hyper-reactivity [17], the role of aspirin in the pathogenesis of cancerassociated VTE and its role for primary VTE prevention in cancer patients should be priorities. Third, patients often make choices that are different from those expected by thrombosis experts. Discrete choice experiments (DCE) are a powerful tool to determine which aspects of antithrombotic therapy are important for patients not only regarding acute, but also for chronic or even lifelong prevention strategies.

Multiple myeloma (MM) is one of the highest-risk cancers for VTE, and treatment with immune-modulatory drugs further increases the risk. Historically, aspirin is used for primary VTE prevention in such patients and is recommended in the guidelines $[18,19]$. Indeed, a Cochrane review found a non-significant $49 \%$ reduction in the risk of VTE with LMWH compared with aspirin(HR, 0.51; 95\% CI,0.22-1.17) [20]. However, recent observational cohorts, including the Melisse study, showed high rates of VTE with current clinical practice [21]. In this setting, formal studies comparing aspirin with anticoagulant drugs are a priority.

\section{Consensus:}

a. There is no clinical need for further studies of aspirin in primary prevention of VTE in orthopaedic surgery

b. The role of aspirin in primary prevention of VTE in cancer patients should be prioritized

c. In patients with multiple myeloma, we need RCTs comparing aspirin with anticoagulant drugs.

d. Studies of aspirin for secondary prevention should focus on patients with low-to-moderate risk of recurrent VTE, more precise prediction tools are needed before such studies can be initiated

e. More data are needed on the aspects of antithrombotic therapy that are important for patients

\section{New Oral Anticoagulants for VTE treatment; which patients, which drug and which dose}

[Y. Whitney Cheung, Thijs E. van Mens, Jeffrey I. Weitz]

Phase III clinical trials have shown that the NOACs, which include dabigatran, rivaroxaban, apixaban and edoxaban, are at least as effective as VKAs for treatment of patients with VTE and are associated with a reduced risk of major bleeding (relative risk [RR], 0.61; 95\% CI, 0.45-0.83) and intracranial bleeding (RR, 0.37; 95\% CI, 0.21-0.68) [1,3,22-26]. Furthermore, rivaroxaban and apixaban can be administered in alloral regimens, which streamline therapy by obviating the need for initial treatment with a parenteral anticoagulant. Therefore, NOACs simplify VTE treatment.

For extended VTE treatment, dabigatran, rivaroxaban and apixaban were superior to placebo for prevention of recurrence and were associated with low rates of major bleeding $[2,3,27]$. Both the prophylactic and the treatment dose of apixaban ( 2.5 and $5 \mathrm{mg}$ twice daily) were compared with placebo for secondary VTE prevention. Both doses reduced the risk of recurrence by a similar extent. Although rates of 
major bleeding were low, there was a trend for less clinically relevant non-major bleeding with the lower dose regimen [2]. When compared with warfarin for extended VTE treatment, dabigatran had similar efficacy but was associated with a $50 \%$ reduction in the rate of major bleeding [27]. Therefore, NOACs are not only useful for initial VTE treatment, but also are effective and safe for extended VTE treatment. Furthermore, at least with apixaban, it is possible to lower the dose after an initial course of full-dose therapy, thereby reducing the risk of bleeding without compromising efficacy.

\subsection{Unmet Clinical Needs and Knowledge Gaps}

\subsubsection{Prevention of VTE}

Patients with cancer are at risk for VTE, particularly when they are receiving treatment with chemotherapy or biological agents [28]. Prophylactic nadroparin or semuloparin have been shown to lower this risk in high-risk cancer patients $[29,30]$. However, the need for daily subcutaneous injections has tempered enthusiasm for primary prophylaxis with heparin derivatives. With oral administration and a low risk of bleeding, the role of the NOACs for primary prophylaxis in high-risk cancer patients requires investigation.

\subsubsection{Treatment of Acute VTE}

The current standard of care for cancer-associated VTE is LMWH. Cancer patients were under-represented in the phase III trials of the NOACs for VTE treatment. Nonetheless, a meta-analysis of the results in such patients suggests that the NOACs are at least as effective as VKAs [31]. Additional studies are needed to determine how the NOACs compare with LMWH in patients with cancer-associated VTE.

\subsubsection{Optimizing Therapy with the NOACs}

Limited information is available on the benefit-risk profile of NOACs in patients at extremes of body weight because few patients under $50 \mathrm{~kg}$ or over $150 \mathrm{~kg}$ were included in the trials. Likewise, it is unknown whether the absorption of the NOACs is impaired after bariatric procedures such as gastric banding or Roux-en-Y gastric bypass. In addition, the benefit-risk profile of the NOACs in patients with a creatinine clearance less than $30 \mathrm{ml} / \mathrm{min}$ is uncertain. Such information is needed to increase the generalizability of use of the NOACs in the spectrum of VTE patients.

\subsubsection{Secondary VTE Prevention}

The prophylactic dose of apixaban was as effective as the treatment dose for secondary VTE prevention [2]. Whether the same is true with the other NOACs deserves investigation.

\subsubsection{Influence of NOACs on the Risk of PTS}

The risk of PTS in DVT patients treated with NOACs is unknown. Because of their more predictable anticoagulant response, NOACs could decrease the risk of PTS compared with VKAs. However, without the anti-inflammatory effects of heparins, all-oral therapy with NOACs could compromise early thrombus resolution. Additional studies are needed to test these possibilities.

Although NOACs do not require monitoring and dose adjustment, assessing their anticoagulant effect or determining plasma drug concentrations can be useful in patients who present with serious bleeding or acute renal impairment, and in those taking concomitant medications that may interact with the NOACs. The NOACs have variable effects on global tests of coagulation, such as the prothrombin time and activated partial thromboplastin time. Therefore, assays that measure drug levels are needed. These may include the dilute thrombin time or ecarin chromogenic assay for measurement of dabigatran concentrations, and antifactor Xa assays for determination of the levels of rivaroxaban, apixaban and edoxaban. Ideally, these tests should be in point-of-care format to simplify rapid clinical decision making.
Consensus:

a. In cancer patients, there is a need for investigation into the role of NOACs for both VTE treatment and for VTE prophylaxis in high-risk patients.

b. The benefit-risk profiles of NOACs in special patient groups, such as patients with extreme bodyweights, patients after bariatric surgery and patients with a creatinine clearance below $30 \mathrm{ml} / \mathrm{min}$, deserves to further investigation

c. Studies are needed to determine the effect of NOACs on the incidence of PTS.

d. To assess the anticoagulant status of patients taking NOACs, pointof-care assays to measure drug concentrations are desirable.

IV. Direct coagulation inhibitors and contact activation inhibitors Bleker]

[David Gailani, Helen Philippou, José W. Govers-Riemslag, Suzanne

\section{Factor XI and Factor XII}

Based on work with animal models [32,33] and epidemiologic data [34], factor XI (FXI) and factor XII (FXII) are now targets for novel antithrombotic agents. Work with rodents, rabbits and primates indicate that contact activation contributes to thrombus propagation $[32,33]$. There is a particularly strong case for a role for FXI in venous thromboembolism (VTE) and stroke in humans [34]. As FXII deficiency does not cause abnormal hemostasis [35], and FXI deficiency is associated with mild bleeding [36], agents targeting these proteins should cause less bleeding than VKAs or NOACs. Several proteins and a few small molecule inhibitors of FXII and FXI, or their active forms FXIIa and FXIa, have undergone preclinical testing [37]. Second generation antisense oligonucleotides (ASOs) that reduce FXI synthesis have been studied in humans [38,39]. Büller et al. compared ASO-mediated reduction of plasma FXI with enoxaparin for thromboprophylaxis in patients undergoing total knee arthroplasty [39]. The highest ASO dose tested reduced plasma FXI levels to 20\% of normal, with some patients having nearly undetectable levels. This group had a markedly lower rate of radiographically detected DVT (4\%) than that in the enoxaparin controls (30\%). Interestingly, the few clots that formed in the high-dose ASO group were very small, suggesting FXI contributes at an early stage of thrombus growth. This challenges the prevailing notion that tissue factor is the major initiator and driver of VTE. Despite reduced FXI levels, ASO-treated patients did not bleed excessively during surgery, and overall bleeding rates were low in both ASO- and enoxaparin-treated patients, consistent with the prediction that targeting the contact pathway will have limited impact on hemostasis.

Based on the encouraging results from this primary prevention trial, workshop participants felt that a phase 3 trial targeting FXI as secondary prevention for VTE should be organized. While the ASO-based strategy could be employed, an oral agent would be preferable. FXIa inhibitors for parenteral administration are entering phase 1 testing, but oral agents are still under development. Given the on-going debate regarding the optimal duration of therapy for unprovoked VTE [40,41], it was felt that there is a need for effective drugs with superior safety profiles to VKAs and NOACs for long-term treatment. There was also enthusiasm for a primary prevention trial using FXIa or FXIIa inhibitors for stroke prevention in patients with atrial fibrillation. It should be possible to superimpose such therapy on standard-of-care without significantly increasing bleeding risk. Patients with severe FXI deficiency with atrial fibrillation tolerate warfarin therapy (target INR 2.0 and 2.5) and FXI-deficient patients have been placed on aspirin and clopidogrel post-myocardial infarction [42].

Members of the workshop felt that contact activation inhibitors should be tested in cancer patients to prevent VTE. Many of these patients are at high risk for both thrombosis and bleeding, so an antithrombotic agent that does not compromise hemostasis would be 
beneficial. Inhibitors of contact activation may also have a role in patients with chronic indwelling catheters for venous access or dialysis to prevent catheter occlusion or catheter-associated thrombosis. In support of this, in recent studies, reducing FXII or FXI levels prolonged the time to occlusion of catheters inserted into rabbit jugular veins [43]. Contact activation inhibitors may turn out to be useful in a variety of settings where blood comes into contact with artificial surfaces. Contact activation is triggered during cardiopulmonary bypass and extracorporeal membrane oxygenation. The recent demonstration that an anti-FXIIa antibody was comparable to heparin in its ability to prevent thrombus formation in rabbits attached to a pediatric ECMO circuit supports the notion that contact activation is a driver of thrombosis in extracorporeal circuits [44]. Given the poor performance of dabigatran in a trial of patients with mechanical heart valves, VKAs remains the only oral agents available for long-term treatment in this setting [45]. While the contribution of the contact system to thrombosis associated with artificial valves is not clear, materials used in heart valves should be tested for their ability to induce contact activation.

As a long-term goal, we need to establish the roles of FXII and FXI in different clinical scenarios to identify which target is best for various indications. In general, FXI may contribute to thrombosis through two pathways; contact activation and thrombin-mediated feedback activation of the intrinsic pathway [37]. However, FXIIa may contribute to thrombosis not only by activating FXIa, but through FXI-independent effects on fibrin clot stability [46]. Furthermore, FXIIa inhibition may more effectively limit prekallikrein activation, and be a better antiinflammatory agent than FXIa inhibitors. The main safety concern with FXIa inhibitors relates to the bleeding diathesis that can occur with severe FXI deficiency. A concern raised with targeting FXII is that it appears to be involved in processes other than thrombus formation. FXIIa inhibitors could, for example, block the contribution of contact activation to fibrinolysis $[47,48]$. However, there are other more potent activators of fibrinolysis in blood, and it is not clear that loss of FXIla activity would produce a clinically significant fibrinolytic defect. Inhibition of FXIIa leads to dose-dependent prevention of clot formation [44].

Finally, we discussed the optimal levels of inhibition for FXI or FXII. Hypothetically, complete inhibition of either target would be associated with relatively few side effects, but there are concerns. Complete neutralization of FXI could produce a bleeding diathesis similar to severe congenital FXI deficiency [36,42]. While the intracranial, retroperitoneal and gastrointestinal bleeding events that complicate therapy with VKAs or NOACs are unlikely to occur with FXI inhibitors (even with complete inhibition), there could be problems with mucosal bleeding, particularly in menstruating woman. Based on ASO data from humans and nonhuman primates [38,49,50], reducing FXI activity to $20 \%$ of normal may strike a reasonable balance between efficacy and bleeding risk. For a FXII inhibitor, there was concern regarding the epidemiologic data indicating an inverse relationship between plasma FXII level and ischemic heart disease $[51,52]$. Interestingly, while the correlation was apparent in two large studies for levels within the broad normal range, patients with severe FXII deficiency were not at higher risk for cardiac disease [52]. Thus, it may be best to have near complete inhibition of FXII to produce an antithrombotic effect.

As we are able to progress towards contact pathway inhibition, it is apparent that we are now entering an era where anticoagulant efficacy may be achieved without the need to balance the induced risk of bleeding associated with other anticoagulant targets.

\section{Consensus:}

a. FXI is a potential factor to inhibit for secondary VTE prevention

b. Phase 3 studies are needed to further investigate the role of inhibition of the contact pathway for secondary VTE prevention

c. The utility of inhibition of the contact pathway in patients with catheters or cancer deserves investigation d. Role of FXII or contact activation in VTE, cardiopulmonary bypass or mechanical valves needs to be determined

e. The optimal FXI or FXII level required to attenuate thrombosis needs to be established

f. FXII has multiple functions including possibly playing a role in fibrinolysis in circumstances of low concentrations of tPA or UPA. Whether FXII plays a role in fibrinolysis in vivo remains to be established.. If FXII does play a role in fibrinolysis then FXII inhibitors should be used in prophylactic settings.

g. Oral inhibitors of the contact system are preferred over parenteral agents

\section{Conservative management of the patient with PTS}

[Arina J. ten Cate-Hoek, Annemieke C. Bouman]

PTS occurs in up to $50 \%$ of patients with deep-vein thrombosis (DVT) [53-55]. PTS decreases quality of life and can lead to chronic ulcers, and its management is costly for the healthcare system [54]. Extensive DVT, recurrent ipsilateral DVT and inadequate early anticoagulation are risk factors for PTS [56,57]. Although studies suggested that early application of elastic compression stockings (ECS) reduced the risk of PTS [53,58], their effectiveness has been called into question by the results of a recent randomized, placebo-controlled trial that showed that compared with sham stockings, ECS did not prevent PTS [59].

The most important drawback for research and progress in clinical care has been the limited correlation between the available diagnostic clinical scales [60]. There are no dedicated prediction scores that incorporate clinical signs as well as established risk factors for PTS. The Villalta score has been branded as the preferred diagnostic tool by the International Society on Thrombosis and Haemostasis (ISTH) [61]. There is however still a need to improve the diagnostic accuracy and the predictive quality of the Villalta score. It has been suggested to use the Villalta score together with a disease specific quality of life score, the Veines-Qol/Sym as the gold standard for the diagnosis of PTS [62].

It was discussed that it might be more efficient to add distinctive and currently missing items such as venous claudication to the Villalta score. Additional items suggested included impaired ankle movement, leg circumference, thrombus extent and biomarkers for PTS. For the late stage follow-up and assessment of clinical change, there is a need to improve the sensitivity to clinical change especially with respect to ulceration.

Workshop participants felt that more evidence is needed on the preventive qualities of compression therapy. The relevance of compression therapy for thrombus resolution and its influence on inflammation, and the consequences on PTS incidence should be further elucidated.

For the (conservative) treatment of PTS, more research into the relevance of compression therapy as a mechanistic component for edema reduction is needed. For the question as to whether compression therapy can be of value for the prevention of PTS, the items that should be addressed were discussed and it was felt that further studies are needed on the value of compression (inelastic or elastic compression), the timing of compression (time to compression, optimal duration of compression), and issues surrounding compliance with therapy. (DCE, education, assisting devices).

Both for the prevention and treatment of PTS, pharmacological treatment such as drugs that increase the vascular integrity, veno-active drugs (VADs), statins, heparins, and anti-inflammatory drugs should be evaluated. Also, NOACs as a potential option for PTS prevention should be investigated. Concern was expressed that pharmacological therapy should not undermine compression therapy.

Biomarkers of inflammation and endothelial activation need to be investigated more rigorously and endothelial activation and permeability were perceived as an important pathway for subsequent inflammatory processes in the perivascular space.

One of the obstacles in advancing PTS research is the lack of an objective diagnostic strategy for establishing the diagnosis of PTS. Until now radiological vein wall abnormalities and valvular abnormalities only show a poor association with clinical symptoms of PTS. With the 
development of more sensitive radiological techniques the assessment of thrombus resolution and associated inflammatory processes of the vein wall in the acute phase may be better visualised and may aid the diagnostic procedure.

a. More evidence is needed about compression therapy

b. Focus should be on pharmacological therapy, but should not undermine the compression therapy

c. NOACs as a potential option for PTS prevention should be investigated

d. Separate prediction scores are needed for prevention and treatment phase, including clinical scores and biomarkers

\section{Proposal for Future Research Directions}

The pleiotropic effects of statins need further research both for the long-term prevention of recurrent VTE, as well as for its possible mitigating effect on the inflammatory response associated with thrombus resolution and subsequent PTS.

Appropriate animal models of VTE and PTS are needed that incorporate processes of ageing and inflammation to further study thrombosisinflammation-crosstalk and to better understand the pathophysiology of both primary and recurrent VTE and PTS.

There is no place for aspirin as secondary preventive treatment except for specific patient categories such as patients with certain solid tumours. This application needs further study.

Further studies should be planned to assess alternative anticoagulant treatment modalities that inhibit the contact pathway i.e. at the level of FXI or FXII, which may attenuate the risk of bleeding.

More research is needed for the assessment of preventive and therapeutic qualities of compression therapy in VTE. The diagnostic procedure needs adaptation and further evaluation. The potential role of VADs, but also of statins, heparins and anti-inflammatory drugs, for the prevention of PTS needs to be elucidated.

\section{Significance of Increased Research}

There are unmet needs concerning the non-invasive management for both (recurrent) VTE and PTS. Reduction of bleeding events associated with anticoagulant treatment will lower the burden of disease and make anticoagulant treatment more accessible for patients at high risk for both VTE and bleeding. A definite target population in need of improved anticoagulant treatment is the population of patients with cancer. Also, long-term anticoagulant treatment for prevention of recurrent VTE will be easier to implement with agents that attenuate thrombosis without affecting hemostatsis. The study of drugs such as aspirin and statins currently in use for other indications and thus directly available may expedite the process. Basic research into the mechanisms of coagulation and inflammation may lead to the development of other more favorable forms of antithrombotic treatment. Prevention of recurrent VTE will decrease the morbidity associated with PTS. Localized processes of endothelial cell adhesion, and inflammation associated with thrombosis need to be unraveled to optimize prevention and treatment of VTE and its complications.

\section{Conflict of Interest Statement}

The following authors have no conflict of interest to report: Harry R. Buller, Arina J. ten Cate-Hoek, Helen Philippou, Annemieke C. Bouman, Y. Whitney Cheung, Thijs E. van Mens, Jose W. Govers-Riemslag, Minka Vries, Suzanne Bleker, Jossi S. Biedermann, S. and Carina M. Stoof.

Karina Meijer has received research fees from Baxter, Bayer and Sanquin, travel support from Baxter, Bayer, Sanquin and Pfizer, and speaker fees from Sanquin and Boehringer Ingelheim.

David Gailani receives consultant fees from: Bayer Pharma AG, Bristol-Myers-Squibb Co., Dyax Corp., Isis Pharmaceuticals, Merck \& Co., Novartis International AG, and Ono Pharmaceuticals.
Dr. Weitz has received consulting fees from Boehringer-Ingelheim, Bayer, Bristol-Myers Squibb, Pfizer, Daiichi Sankyo, Janssen, ISIS Pharmaceuticals and Portola.

\section{References}

[1] N. van Es, M. Coppens, S. Schulman, S. Middeldorp, H.R. Buller, Direct oral anticoagulants compared with vitamin $\mathrm{K}$ antagonists for acute venous thromboembolism: evidence from phase 3 trials, Blood 124 (2014) 1968-1975.

[2] G. Agnelli, H.R. Buller, A. Cohen, M. Curto, A.S. Gallus, M. Johnson, et al., Apixaban for extended treatment of venous thromboembolism, N. Engl. J. Med. 368 (2013) 699-708.

[3] R. Bauersachs, S.D. Berkowitz, B. Brenner, H.R. Buller, H. Decousus, A.S. Gallus, et al., Oral rivaroxaban for symptomatic venous thromboembolism, N. Engl. J. Med. 363 (2010) 2499-2510.

[4] M. Takemoto, J.K. Liao, Pleiotropic effects of 3-hydroxy-3-methylglutaryl coenzyme a reductase inhibitors, Arterioscler. Thromb. Vasc. Biol. 21 (2001) 1712-1719.

[5] A. Undas, K.E. Brummel-Ziedins, K.G. Mann, Anticoagulant effects of statins and their clinical implications, Thromb. Haemost. 111 (2014) 392-400.

[6] A. Squizzato, M. Galli, E. Romualdi, F. Dentali, P.W. Kamphuisen, L. Guasti, et al., Statins, fibrates, and venous thromboembolism: a meta-analysis, Eur. Heart J. 31 (2010) 1248-1256.

[7] S. Biere-Rafi, B.A. Hutten, A. Squizzato, W. Ageno, P.C. Souverein, A. de Boer, et al., Statin treatment and the risk of recurrent pulmonary embolism, Eur. Heart J. 34 (2013) 1800-1806.

[8] C.D. Nguyen, C. Andersson, T.B. Jensen, A. Gjesing, A.M. Schjerning Olsen, C. Malta Hansen, et al., Statin treatment and risk of recurrent venous thromboembolism: a nationwide cohort study, BMJ Open 3 (2013) e003135.

[9] M. Schmidt, S.C. Cannegieter, S.A. Johannesdottir, O.M. Dekkers, E. Horvath-Puho, H.T. Sorensen, Statin use and venous thromboembolism recurrence: a combined nationwide cohort and nested case-control study, J. Thromb. Haemost. 12 (2014) 1207-1215.

[10] R.J. Glynn, E. Danielson, F.A. Fonseca, J. Genest, A.M. Gotto Jr., J.J. Kastelein, et al., A randomized trial of rosuvastatin in the prevention of venous thromboembolism, N. Engl. J. Med. 360 (2009) 1851-1861.

[11] P.M. Ridker, T.F. Luscher, Anti-inflammatory therapies for cardiovascular disease, Eur. Heart J. 35 (2014) 1782-1791.

[12] Prevention of pulmonary embolism and deep vein thrombosis with low dose aspirin: Pulmonary Embolism Prevention (PEP) trial, Lancet 355 (2000) 1295-1302.

[13] G.H. Guyatt, E.A. Akl, M. Crowther, D.D. Gutterman, H.J. Schuunemann, Executive summary: Antithrombotic Therapy and Prevention of Thrombosis, 9th ed: American College of Chest Physicians Evidence-Based Clinical Practice Guidelines, Chest. 141 (2012) 7S-47S.

[14] D.R. Anderson, M.J. Dunbar, E.R. Bohm, E. Belzile, S.R. Kahn, D. Zukor, et al., Aspirin versus low-molecular-weight heparin for extended venous thromboembolism prophylaxis after total hip arthroplasty: a randomized trial, Ann. Intern. Med. 158 (2013) 800-806.

[15] J. Simes, C. Becattini, G. Agnelli, J.W. Eikelboom, A.C. Kirby, R. Mister, et al., Aspirin for the prevention of recurrent venous thromboembolism: the INSPIRE collaboration, Circulation 130 (2014) 1062-1071.

[16] L.A. Castellucci, C. Cameron, G. Le Gal, M.A. Rodger, D. Coyle, P.S. Wells, et al., Efficacy and safety outcomes of oral anticoagulants and antiplatelet drugs in the secondary prevention of venous thromboembolism: systematic review and network meta-analysis, BMJ Clin. Res. Ed. 347 (2013) f5133.

[17] N. van Es, A. Sturk, S. Middeldorp, R. Nieuwland, Effects of cancer on platelets, Semin. Oncol. 41 (2014) 311-318.

[18] G.H. Lyman, K. Bohlke, A.A. Khorana, N.M. Kuderer, A.Y Lee, J.I. Arcelus, et al, Venous thromboembolism prophylaxis and treatment in patients with cancer: american society of clinical oncology clinical practice guideline update 2014, J. Clin. Oncol. Off. J. Am. Soc. Clin. Oncol. 33 (2015) 654-656.

[19] S. Zweegman, H.M. Lokhorts, M.D. Levin, E. de Waal, G.M.J. Bos, M.J. Kersten, et al., Richtlijnen behandeling multiple myeloom, Ned. Tijdschr. Hematol. (2012) 300-320.

[20] M. Di Nisio, E. Porreca, H.M. Otten, A.W. Rutjes, Primary prophylaxis for venous thromboembolism in ambulatory cancer patients receiving chemotherapy, Cochrane Database Syst. Rev. 8 (2014) Cd008500.

[21] X. Leleu, P. Rodon, C. Hulin, L. Daley, C. Dauriac, M. Hacini, et al., MELISSE, a large multicentric observational study to determine risk factors of venous thromboembolism in patients with multiple myeloma treated with immunomodulatory drugs, Thromb. Haemost. 110 (2013) 844-851.

[22] G. Agnelli, H.R. Buller, A. Cohen, M. Curto, A.S. Gallus, M. Johnson, et al., Oral apixaban for the treatment of acute venous thromboembolism, N. Engl. J. Med. 369 (2013) 799-808.

[23] H.R. Buller, H. Decousus, M.A. Grosso, M. Mercuri, S. Middeldorp, M.H. Prins, et al. Edoxaban versus warfarin for the treatment of symptomatic venous thromboembolism, N. Engl. J. Med. 369 (2013) 1406-1415

[24] H.R. Buller, M.H. Prins, A.W. Lensin, H. Decousus, B.F. Jacobson, E. Minar, et al., Oral rivaroxaban for the treatment of symptomatic pulmonary embolism, N. Engl. J. Med. 366 (2012) 1287-1297.

[25] S. Schulman, A.K. Kakkar, S. Schellong, S.Z. Goldhaber, E. Henry, P. Mismetti, et al., A randomized trial of dabigatran versus warfarin in the treatment of acute venous thromboembolism (RE-COVER II), ASH Annual Meeting Abstracts, 2011.

[26] S. Schulman, C. Kearon, A.K. Kakkar, P. Mismetti, S. Schellong, H. Eriksson, et al., Dabigatran versus warfarin in the treatment of acute venous thromboembolism, N. Engl. J. Med. 361 (2009) 2342-2352. 
[27] S. Schulman, C. Kearon, A.K. Kakkar, S. Schellong, H. Eriksson, D. Baanstra, et al., Extended use of dabigatran, warfarin, or placebo in venous thromboembolism, $\mathrm{N}$. Engl. J. Med. 368 (2013) 709-718.

[28] J.A. Heit, M.D. Silverstein, D.N. Mohr, T.M. Petterson, C.M. Lohse, W.M. O'Fallon, et al., The epidemiology of venous thromboembolism in the community, Thromb. Haemost. 86 (2001) 452-463.

[29] G. Agnelli, D.J. George, A.K. Kakkar, W. Fisher, M.R. Lassen, P. Mismetti, et al., Semuloparin for thromboprophylaxis in patients receiving chemotherapy for cancer, N. Engl. J. Med. 366 (2012) 601-609.

[30] A.T. Cohen, M.M. Gurwith, M. Dobromirski, Thromboprophylaxis in non-surgical cancer patients, Thromb. Res. 129 (Suppl. 1) (2012) S137-S145.

[31] T.B. Larsen, P.B. Nielsen, F. Skjoth, L.H. Rasmussen, G.Y. Lip, Non-vitamin K antagonist oral anticoagulants and the treatment of venous thromboembolism in cancer patients: a semi systematic review and meta-analysis of safety and efficacy outcomes, PLoS One 9 (2014) e114445.

[32] T. Renne, The procoagulant and proinflammatory plasma contact system, Semin. Immunopathol. 34 (2012) 31-41.

[33] M.L. van Montfoort, J.C. Meijers, Recent insights into the role of the contact pathway in thrombo-inflammatory disorders, Hematology Am. Soc. Hematol. Educ. Program 2014 (2014) 60-65.

[34] N.S. Key, Epidemiologic and clinical data linking factors XI and XII to thrombosis, Hematology Am. Soc. Hematol. Educ. Program 2014 (2014) 66-70.

[35] A.H. Schmaier, Physiologic activities of the contact activation system, Thromb. Res. 133 (Suppl. 1) (2014) S41-S44.

[36] S. Duga, O. Salomon, Congenital factor XI deficiency: an update, Semin. Thromb. Hemost. 39 (2013) 621-631.

[37] D. Gailani, Future prospects for contact factors as therapeutic targets, Hematology Am. Soc. Hematol. Educ. Program 2014 (2014) 52-59.

[38] H.R. Buller, C. Bethune, S. Bhanot, D. Gailani, B.P. Monia, G.E. Raskob, et al., Factor XI antisense oligonucleotide for prevention of venous thrombosis, N. Engl. J. Med. 372 (2015) 232-240.

[39] Q. Liu, C. Bethune, E. Dessouki, J. Grundy, B.P. Monia, S. Bhanot, ISIS-FXIRx, A Novel and Specific Antisense Inhibitor of Factor XI, Caused Significant Reduction in FXI Antigen and Activity and Increased aPTT without Causing Bleeding in Healthy Volunteers, Blood 118 (2011) (abstract 209).

[40] C. Kearon, E.A. Akl, Duration of anticoagulant therapy for deep vein thrombosis and pulmonary embolism, Blood 123 (2014) 1794-1801.

[41] S. Schulman, J. Douketis, Secondary prevention of venous thromboembolism, BMJ Clin. Res. Ed. 347 (2013) f5440.

[42] O. Salomon, U. Seligsohn, New observations on factor XI deficiency, Haemophilia 10 (Suppl. 4) (2004) 184-187.

[43] J.W. Yau, P. Liao, J.C. Fredenburgh, A.R. Stafford, A.S. Revenko, B.P. Monia, et al., Selective depletion of factor XI or factor XII with antisense oligonucleotides attenuates catheter thrombosis in rabbits, Blood 123 (2014) 2102-2107.

[44] M. Larsson, V. Rayzman, M.W. Nolte, K.F. Nickel, J. Bjorkqvist, A. Jamsa, et al., A factor XIla inhibitory antibody provides thromboprotection in extracorporeal circulation without increasing bleeding risk, Sci. Transl. Med. 6 (2014) 222 ra17.

[45] J.W. Eikelboom, S.J. Connolly, M. Brueckmann, C.B. Granger, A.P. Kappetein, M.J. Mack, et al., Dabigatran versus warfarin in patients with mechanical heart valves, N. Engl. J. Med. 369 (2013) 1206-1214.
[46] J. Konings, J.W. Govers-Riemslag, H. Philippou, N.J. Mutch, J.I. Borissoff, P. Allan, et al., Factor XIla regulates the structure of the fibrin clot independently of thrombin generation through direct interaction with fibrin, Blood 118 (2011) 3942-3951.

[47] C. Kluft, G. Dooijewaard, J.J. Emeis, Role of the contact system in fibrinolysis, Semin. Thromb. Hemost. 13 (1987) 50-68.

[48] A. Tanaka, Y. Suzuki, K. Sugihara, N. Kanayama, T. Urano, Inactivation of plasminogen activator inhibitor type 1 by activated factor XII plays a role in the enhancement of fibrinolysis by contact factors in-vitro, Life Sci. 85 (2009) 220-225.

[49] J.R. Crosby, U. Marzec, A.S. Revenko, C. Zhao, D. Gao, A. Matafonov, et al., Antithrombotic effect of antisense factor XI oligonucleotide treatment in primates, Arterioscler. Thromb. Vasc. Biol. 33 (2013) 1670-1678.

[50] H.S. Younis, J. Crosby, J.I. Huh, H.S. Lee, S. Rime, B. Monia, et al., Antisense inhibition of coagulation factor XI prolongs APTT without increased bleeding risk in cynomolgus monkeys, Blood 119 (2012) 2401-2408.

[51] C.J. Doggen, F.R. Rosendaal, J.C. Meijers, Levels of intrinsic coagulation factors and the risk of myocardial infarction among men: Opposite and synergistic effects of factors XI and XII, Blood 108 (2006) 4045-4051.

[52] G. Endler, C. Marsik, B. Jilma, T. Schickbauer, P. Quehenberger, C. Mannhalter, Evidence of a U-shaped association between factor XII activity and overall survival, J. Thromb. Haemost. 5 (2007) 1143-1148.

[53] D.P. Brandjes, H.R. Buller, H. Heijboer, M.V. Huisman, M. de Rijk, H. Jagt, et al., Randomised trial of effect of compression stockings in patients with symptomatic proximal-vein thrombosis, Lancet 349 (1997) 759-762.

[54] S.R. Kahn, I. Shrier, J.A. Julian, T. Ducruet, L. Arsenault, M.J. Miron, et al., Determinants and time course of the postthrombotic syndrome after acute deep venous thrombosis, Ann. Intern. Med. 149 (2008) 698-707.

[55] P. Prandoni, S. Villalta, P. Bagatella, L. Rossi, A. Marchiori, A. Piccioli, et al., The clinical course of deep-vein thrombosis. Prospective long-term follow-up of 528 symptomatic patients, Haematologica 82 (1997) 423-428.

[56] R.S. Chitsike, M.A. Rodger, M.J. Kovacs, M.T. Betancourt, P.S. Wells, D.R. Anderson, et al., Risk of post-thrombotic syndrome after subtherapeutic warfarin anticoagulation for a first unprovoked deep vein thrombosis: results from the REVERSE study, J. Thromb. Haemost. 10 (2012) 2039-2044.

[57] C.J. van Dongen, P. Prandoni, M. Frulla, A. Marchiori, M.H. Prins, B.A. Hutten, Relation between quality of anticoagulant treatment and the development of the postthrombotic syndrome, J. Thromb. Haemost. 3 (2005) 939-942.

[58] P. Prandoni, A.W. Lensing, M.H. Prins, M. Frulla, A. Marchiori, E. Bernardi, et al., Below-knee elastic compression stockings to prevent the post-thrombotic syndrome: a randomized, controlled trial, Ann. Intern. Med. 141 (2004) 249-256.

[59] S.R. Kahn, S. Shapiro, P.S. Wells, M.A. Rodger, M.J. Kovacs, D.R. Anderson, et al, Compression stockings to prevent post-thrombotic syndrome: a randomised placebo-controlled trial, Lancet 383 (2014) 880-888.

[60] D.N. Kolbach, H.A. Neumann, M.H. Prins, Definition of the post-thrombotic syndrome, differences between existing classifications, Eur. J. Vasc. Endovasc. Surg. 30 (2005) 404-414.

[61] S.R. Kahn, H. Partsch, S. Vedantham, P. Prandoni, C. Kearon, Definition of postthrombotic syndrome of the leg for use in clinical investigations: a recommendation for standardization, J. Thromb. Haemost. 7 (2009) 879-883.

[62] A. Soosainathan, H.M. Moore, M.S. Gohel, A.H. Davies, Scoring systems for the post-thrombotic syndrome, J. Vasc. Surg. 57 (2013) 254-261. 